\title{
A PROOF OF THE FULL MARIÑO-VAFA CONJECTURE
}

\author{
JIAN ZHOU
}

\begin{abstract}
We present a proof of the full Mariño-Vafa Conjecture that identifies certain open string invariants of the resolved conifold with the Chern-Simons knot invariant of the unknot, i.e. the quantum dimensions. This proof is based on earlier works $[9,14,10]$ that culminate in a mathematical theory of the topological vertex [8].
\end{abstract}

\section{Introduction}

The duality between topological string theory and Chern-Simons theory is one of many important examples of string duality. Mathematically, this duality predicts an amazing connection between Gromov-Witten theory and link invariants. Such predictions have inspired many mathematical results that verify this connection. In this short note we will present a proof of the full Mariño-Vafa Conjecture [13] that identifies certain open string invariants of the resolved conifold with the Chern-Simons knot invariant of the unknot, i.e. the quantum dimensions, based on some of such results.

Witten [17] proposed that the large N expansion of Chern-Simons link invariants [16] of $S^{3}$ can be identified with the genus expansion of open string invariants of the deformed conifold $T^{*} S^{3}$, which counts holomorphic maps from Riemann surfaces with boundaries to $T^{*} S^{3}$, with boundary components mapped to the Lagrangian submanifold $S^{3}$. Gopakumar and Vafa [3] proposed that after summing over the number of boundary components, one gets from the large $N$ expansion of the 3-manifold invariant of $S^{3}$ the genus expansion of closed string invariants of the resolved conifold. Ooguri and Vafa [15] extended this and proposed that the large $N$ expansion of link invariants corresponds to open string invariants of the resolved conifold, associated with some Lagrangian submanifolds corresponding to the link. Mariño and Vafa [13] extended this further by considering framed knots. Later, the duality has been extended to other local Calabi-Yau geometries [2] and a formalism called the topological vertex [1] has been developed to compute open and closed string invariants of local toric Calabi-Yau geometries, in terms of quantities related to the link invariants of the Hopf link. There are many related works in the physics literature. More recently, duality between topological strings and link homology [7] has been proposed $[4,5]$.

Many predictions in the physics literature have been made mathematically rigorous. By comparing with the formal localization calculations performed for the resolved conifold by Katz and Liu [6], Mariño and Vafa [13] conjectured a closed formula for some Hodge integrals. This formula for Hodge integrals has been proved $[9,14]$ and generalized $[18,10,8]$. Such formulas turns out to be important for the mathematical calculations of open and closed string invariants of local Calabi-Yau geometries [19,

Received by the editors April 8, 2010. 
8]. Because mathematically the open string invariants is very difficult to define in symplectic geometry, relative moduli spaces in algebraic geometry have been used to bypass this difficulty $[11,8]$, and a mathematical theory of the topological vertex has been developed [8].

Despite many progresses in the mathematical literature, the full Mariño-Vafa Conjecture has remained unproved for a long time. This is partly because one has to first give a precise formulation of this conjecture. For a long time the author has attempted to give a formulation for the inner brane case without success. In this work we will present a formulation in Theorem 3.1 for the outer brane cases. Our proof of the full Mariño-Vafa Conjecture starts with the computations of the open string invariants of the resolved conifold by the mathematical theory of the topological vertex [8]. Such computations involve complicated summations over two partitions, they are difficult to carry out directly. We will use a simplifying trick discovered in an earlier work $[21]$.

\section{Acknowledgements}

This research is partially supported by two NSFC grants (10425101 and 10631050) and a 973 project grant NKBRPC (2006cB805905). The author thanks an anonymous referee for a careful reading of the paper.

\section{Preliminaries}

In this section we express the quantum dimensions as specializations of Schur functions. We use [12] for references on symmetric functions.

2.1. Symmetric functions. The Newton functions $\left\{p_{\mu}\right\}$ and the Schur functions $\left\{s_{\nu}\right\}$ form additive bases of $\Lambda$, the space of symmetric functions. They are related by the character values:

$$
p_{\mu}=\sum_{\nu} \chi_{\nu}(\mu) s_{\nu}, \quad s_{\nu}=\sum_{\mu} \frac{\chi_{\nu}(\mu)}{z_{\mu}} p_{\mu} .
$$

Here $\mu=\left(\mu_{1}, \ldots, \mu_{l}\right)$ is a partition,

$$
z_{\mu}=\prod_{i=1}^{l(\mu)} \mu_{i} \cdot|\operatorname{Aut}(\mu)|
$$

and $\chi_{\mu}$ denotes the character of the irreducible representation of $S_{|\mu|},|\mu|=\sum_{i} \mu_{i}$, indexed by $\mu$, and $\chi_{\mu}(\nu)$ denotes the value of $\chi_{\mu}$ on the conjugacy class of $S_{|\mu|}$ indexed by $\nu$. The character values satisfy the following orthogonality relations:

$$
\sum_{\nu} \frac{1}{z_{\nu}} \chi_{\mu}(\nu) \chi_{\eta}(\nu)=\delta_{\mu, \eta}, \quad \sum_{\mu} \chi_{\mu}(\xi) \chi_{\mu}(\phi)=\delta_{\xi, \phi} z_{\xi} .
$$

Let $\mathbf{x}=\left(x_{1}, x_{2}, \ldots\right)$ and $\mathbf{y}=\left(y_{1}, y_{2}, \ldots\right)$. Then by (1) and (3) one has

$$
\sum_{\mu} s_{\mu}(\mathbf{x}) s_{\mu}(\mathbf{y})=\sum_{\nu} \frac{1}{z_{\nu}} p_{\nu}(\mathbf{x}) p_{\nu}(\mathbf{y})=\exp \sum_{n=1}^{\infty} \frac{1}{n} p_{n}(\mathbf{x}) p_{n}(\mathbf{y}) .
$$


2.2. Scalar product and vacuum expectation values. There is a natural scalar product on $\Lambda$ such that:

$$
\left\langle p_{\mu}, p_{\nu}\right\rangle=\delta_{\mu, \nu} z_{\mu}, \quad\left\langle s_{\mu}, s_{\nu}\right\rangle=\delta_{\mu, \nu} .
$$

In particular,

$$
\left\langle p_{\mu}, s_{\nu}\right\rangle=\chi_{\nu}(\mu)
$$

Following physical notations, we will write $|\mu\rangle:=s_{\mu}$, and write the inner product $\left\langle A s_{\mu}, s_{\nu}\right\rangle$ for some linear operator $A: \Lambda \rightarrow \Lambda$ as $\langle\nu|A| \mu\rangle$. In particular,

$$
\langle A\rangle:=\langle 0|A| 0\rangle
$$

is called the vacuum expectation value of the operator $A$, where $|0\rangle=1 \in \Lambda$.

2.3. Creators and annihilators. On $\Lambda$ one can introduce the following operators for nonzero integers $n$ :

$$
\beta_{n}: \Lambda \rightarrow \Lambda, \quad \beta_{n}(f)= \begin{cases}p_{-n} \cdot f, & n<0, \\ n \frac{\partial}{\partial p_{n}} f, & n>0 .\end{cases}
$$

These operators satisfy the following Heisenberg commutation relations:

$$
\left[\beta_{m}, \beta_{n}\right]=m \delta_{m,-n} \operatorname{Id}_{\Lambda} .
$$

For a partition $\mu=\left(\mu_{1}, \ldots, \mu_{l}\right)$, we write

$$
\beta_{\mu}=\prod_{i=1}^{l} \beta_{\mu_{i}}, \quad \beta_{-\mu}=\prod_{i=1}^{l} \beta_{-\mu_{i}} .
$$

One clearly has

$$
\beta_{n}|0\rangle=0, \quad n>0 ; \quad \beta_{-\mu}|0\rangle=p_{\mu} .
$$

Hence the operators $\left\{\beta_{n}\right\}_{n>0}$ are called the annihilators, and the operators $\left\{\beta_{-n}\right\}_{n>0}$ are called the creators.

It is easy to see that

$$
\left\langle\beta_{\mu} \beta_{-\nu}\right\rangle=\delta_{\mu, \nu} z_{\mu} .
$$

This is called the Wick formula. It follows from this identity that

$$
\left\langle\exp \left(\sum_{n=1} \frac{a_{n}}{n} \beta_{n}\right) \cdot \exp \left(\sum_{n=1}^{\infty} \frac{b_{n}}{n} \beta_{-n}\right)\right\rangle=\exp \sum_{n=1}^{\infty} \frac{a_{n} b_{n}}{n} .
$$

We will understand $\Lambda$ as the space of symmetric functions in $\mathbf{x}$. Then we have

$$
\exp \left(\sum_{n=1}^{\infty} \frac{1}{n} p_{n}(\mathbf{y}) \beta_{-n}\right)|0\rangle=\sum_{\nu} \frac{1}{z_{\nu}} p_{\nu}(\mathbf{y}) \beta_{-\nu}|0\rangle .
$$

Now we apply (4) to get:

$$
\exp \left(\sum_{n=1}^{\infty} \frac{1}{n} p_{n}(\mathbf{y}) \beta_{-n}\right)|0\rangle=\sum_{\mu} s_{\mu}(\mathbf{y})|\mu\rangle .
$$


2.4. Involution. One can define an involution $\omega: \Lambda \rightarrow \Lambda$ by

$$
\omega\left(p_{n}\right)=(-1)^{n-1} p_{n}
$$

One has

$$
\omega\left(s_{\mu}\right)=s_{\mu^{t}} .
$$

Apply $\omega$ on both sides of (14):

$$
\exp \left(\sum_{n=1}^{\infty} \frac{1}{n}(-1)^{n-1} p_{n}(\mathbf{y}) \beta_{-n}\right)|0\rangle=\sum_{\mu} s_{\mu^{t}}(\mathbf{y})|\mu\rangle .
$$

2.5. Cut-and-join operator. Another useful operator on $\Lambda$ is the cut-and-join operator

$$
\begin{aligned}
K: & =\frac{1}{2} \sum_{i, j=1}^{\infty}\left(p_{i+j} i j \frac{\partial^{2}}{\partial p_{i} \partial p_{j}}+p_{i} p_{j}(i+j) \frac{\partial}{\partial p_{i+j}}\right) \\
& =\frac{1}{2} \sum_{i, j=1}^{\infty}\left(\beta_{-(i+j)} \beta_{i} \beta_{j}+\beta_{-i} \beta_{-j} \beta_{i+j}\right) .
\end{aligned}
$$

The Schur functions are eigenvectors of this operator:

$$
K|\mu\rangle=\frac{1}{2} \kappa_{\mu}|\mu\rangle .
$$

2.6. Specializations of symmetric functions. When $\mathbf{y}=q^{\rho}:=\left(q^{-1 / 2}, q^{-3 / 2}, \ldots\right)$, it is easy to see that

$$
p_{n}\left(q^{\rho}\right)=\frac{1}{q^{n / 2}-q^{-n / 2}}=\frac{1}{[n]} .
$$

Here we use the notation $[n]:=q^{n / 2}-q^{-n / 2}$. It is a very interesting fact that

$$
s_{\mu}\left(q^{\rho}\right)=\frac{q^{\kappa_{\mu} / 4}}{\prod_{x \in \mu}[h(x)]},
$$

where $\kappa_{\mu}=\sum_{i=1}^{l(\mu)} \mu_{i}\left(\mu_{i}-2 i+1\right)$. In other words, when $p_{n}(\mathbf{y})=\frac{1}{[n]}$ for all $n \geq 1$, one has

$$
s_{\mu}(\mathbf{y})=\frac{q^{\kappa_{\mu} / 4}}{\prod_{x \in \mu}[h(x)]} .
$$

By (14),

$$
\exp \left(\sum_{n=1}^{\infty} \frac{1}{n[n]} \beta_{-n}\right)|0\rangle=\sum_{\mu} \frac{q^{\kappa_{\mu} / 4}}{\prod_{x \in \mu}[h(x)]}|\mu\rangle .
$$

We will use another useful specialization. When

$$
p_{n}(\mathbf{y})=\frac{a^{n}-b^{n}}{1-q^{n}}
$$

for $n \geq 1$, one has

$$
s_{\mu}(\mathbf{y})=q^{n(\mu)} \prod_{x \in \mu} \frac{a-b q^{c(x)}}{1-q^{h(x)}} .
$$


Here we represent a partition $\mu$ by its Young diagram, and then $\mu^{t}$ denotes the partition corresponding to the transposed Young diagram of $\mu$. Define

$$
n(\mu)=\sum_{i=1}^{l(\mu)}(i-1) \mu_{i}=\sum_{i=1}^{l\left(\mu^{t}\right)}\left(\begin{array}{c}
\mu_{i}^{t} \\
2
\end{array}\right) .
$$

For a box $x \in \mu$ at the $i$-row and $j$-th column, its content and hook length are defined by:

$$
c(x)=j-i, \quad h(x)=\mu_{i}+\mu_{j}^{t}-i-j+1,
$$

respectively. The following identities hold:

$$
\begin{aligned}
& \sum_{x \in \mu} h(x)=n(\mu)+n\left(\mu^{t}\right)+|\mu|, \\
& \sum_{x \in \mu} c(x)=n\left(\mu^{t}\right)-n(\mu)=\frac{1}{2} \kappa_{\mu} .
\end{aligned}
$$

With these facts, one can easily get the following

Lemma 2.1. If for $n \geq 1$ one has

$$
p_{n}(\mathbf{y})=\frac{1-e^{-n t}}{[n]},
$$

then one has:

$$
s_{\mu}(\mathbf{y})=e^{-|\mu| t / 2} \prod_{x \in \mu} \frac{[c(x)]_{e^{t}}}{[h(x)]}
$$

where

$$
[n]_{e^{t}}=e^{t / 2} q^{n / 2}-e^{-t / 2} q^{-n / 2} .
$$

2.7. Relationship with the large $\mathbf{N}$ quantum dimension. The colored large $\mathrm{N}$ HOMFLY polynomials of the unknot are given by the quantum dimension given as follows $[13,(5.4)]$ :

$$
\operatorname{dim}_{q} R_{\mu}=\prod_{1 \leq i<j \leq l(\mu)} \frac{\left[\mu_{i}-\mu_{j}+j-i\right]}{[j-i]} \cdot \prod_{i=1}^{l(\mu)} \frac{\prod_{j=1}^{\mu_{i}}[j-i]_{e^{t}}}{\prod_{j=1}^{\mu_{i}}[j-i+l(\mu)]} .
$$

By [12, §1.1, Example 1],

$$
\begin{aligned}
& \sum_{x \in \mu} t^{h(x)}+\sum_{1 \leq i<j \leq l(\mu)} t^{\mu_{i}-\mu_{j}+j-i} \\
= & \sum_{i=1}^{l(\mu)} \sum_{j=1}^{\mu_{i}-i+l(\mu)} t^{j}=\sum_{j=1}^{\mu_{i}} t^{j-i+l(\mu)}+\sum_{1 \leq i<j \leq l(\mu)} t^{j-i},
\end{aligned}
$$

therefore,

$$
\operatorname{dim}_{q} R_{\mu}=\prod_{x \in \mu} \frac{[c(x)]_{e^{t}}}{[h(x)]} .
$$

By combining (14), (17), (29), (30) with (33), we get: 
Proposition 2.1. The following identities hold:

$$
\begin{aligned}
& \exp \left(\sum_{n=1}^{\infty} \frac{1-e^{-n t}}{n[n]} \beta_{-n}\right)|0\rangle=\sum_{\mu} e^{-|\mu| t / 2} \cdot \operatorname{dim}_{q} R_{\mu} \cdot|\mu\rangle, \\
& \exp \left(\sum_{n=1}^{\infty}(-1)^{n-1} \frac{1-e^{-n t}}{n[n]} \beta_{-n}\right)|0\rangle=\sum_{\mu} e^{-|\mu| t / 2} \cdot \operatorname{dim}_{q} R_{\mu^{t}} \cdot|\mu\rangle .
\end{aligned}
$$

\section{Proof of the Full Mariño-Vafa Conjecture}

In $\S 3.1$ we first recall some results in [21]. Then we prove the full Mariño-Vafa Conjecture in $\S 3.2$.

3.1. Open string amplitudes with one outer brane. We compute the open string amplitude for the resolved conifold with one outer brane and framing $a$ by the theory of the topological vertex $[1,8]$. There are two possibilities, corresponding to two different toric diagrams as follows:

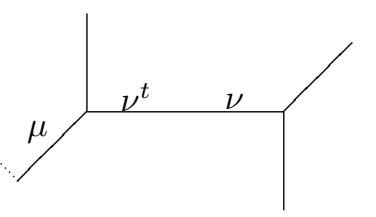

(a)

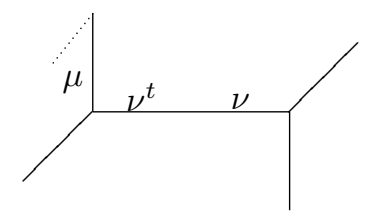

(b)

Figure 1.

For Figure 1(a) we have

$$
Z^{(a)}(\lambda ; t ; \mathbf{p})=\sum_{\mu, \nu, \eta} q^{a \kappa_{\mu} / 2} C_{(0), \mu, \nu^{t}}(q) \cdot Q^{|\nu|} \cdot C_{\nu,(0),(0)}(q) \cdot \frac{\chi_{\mu}(\eta)}{z_{\eta} \sqrt{-1}^{l(\eta)}} p_{\eta}(\mathbf{x}),
$$

where $q=e^{\sqrt{-1} \lambda}$ and $Q=-e^{-t}$. (In physics literature $q=e^{\lambda}$ then one does not need $\sqrt{-1}^{l(\eta)}$ on the right-hand side.) For Figure 1(b) we have

$$
\tilde{Z}^{(a)}(\lambda ; t ; \mathbf{p})=\sum_{\mu, \nu, \eta} q^{a \kappa_{\mu} / 2} C_{\mu,(0), \nu^{t}}(q) \cdot Q^{|\nu|} \cdot C_{\nu,(0),(0)}(q) \cdot \frac{\chi_{\mu}(\eta)}{z_{\eta} \sqrt{-1}^{l(\eta)}} p_{\eta}(\mathbf{x}) .
$$

The topological vertices here can be rewritten as follows (cf [20]):

$$
C_{(0), \mu, \nu^{t}}(q)=q^{-\kappa_{\nu} / 2} W_{\mu, \nu}(q), \quad C_{\mu,(0), \nu^{t}}(q)=q^{\kappa_{\mu} / 2} W_{\nu^{t}, \mu^{t}}(q),
$$

so we have

$$
Z^{(a)}(\lambda ; t ; \mathbf{p})=\sum_{\mu, \nu, \eta} q^{a \kappa_{\mu} / 2} W_{\mu, \nu}(q) q^{-\kappa_{\nu} / 2} Q^{|\nu|} W_{\nu}(q) \cdot \frac{\chi_{\mu}(\eta)}{z_{\eta} \sqrt{-1}^{l(\eta)}} p_{\eta}(\mathbf{x}),
$$

and

$$
\tilde{Z}^{(a)}(\lambda ; t ; \mathbf{p})=\sum_{\mu, \nu, \eta} q^{(a+1) \kappa_{\mu} / 2} W_{\mu^{t}, \nu^{t}}(q) Q^{|\nu|} W_{\nu}(q) \cdot \frac{\chi_{\mu}(\eta)}{z_{\eta} \sqrt{-1}^{l(\eta)}} p_{\eta}(\mathbf{x}) .
$$


The normalized open string amplitudes are defined by:

$$
\hat{Z}^{(a)}(\lambda ; t ; \mathbf{p})=\frac{Z^{(a)}(\lambda ; t ; \mathbf{p})}{\left.Z^{(a)}(\lambda ; t ; \mathbf{p})\right|_{\mathbf{p}=\mathbf{0}}}, \quad \hat{\tilde{Z}}^{(a)}(\lambda ; t ; \mathbf{p})=\frac{\tilde{Z}^{(a)}(\lambda ; t ; \mathbf{p})}{\left.\tilde{Z}^{(a)}(\lambda ; t ; \mathbf{p})\right|_{\mathbf{p}=\mathbf{0}}} .
$$

In [21] we have proved the following:

Proposition 3.1. The normalized open string amplitude of the resolved conifold with one outer brane as in Figure 1(a) and framing $a \in \mathbb{Z}$ is given by:

$$
\hat{Z}^{(a)}(\lambda ; t ; \mathbf{p})=\left\langle\exp \left(\sum_{n=1}^{\infty} \frac{p_{n}(\mathbf{x})}{n \sqrt{-1}} \beta_{n}\right) q^{(a+1) K} \exp \left(\sum_{n=1}^{\infty}\left(\frac{(-1)^{n-1}}{[n]}+\frac{Q^{n}}{[n]}\right) \frac{\beta_{-n}}{n}\right)\right\rangle .
$$

Recall the following identities proved in [20]:

$$
W_{\mu^{t}, \nu^{t}}(q)=(-1)^{|\mu|+|\nu|} W_{\mu, \nu}\left(q^{-1}\right),
$$

and

$$
W_{\nu}(q)=(-1)^{|\nu|} q^{\kappa_{\nu} / 2} W_{\nu}\left(q^{-1}\right)
$$

So we have

$$
\begin{aligned}
& \tilde{Z}^{(a)}(\lambda ; t ; \mathbf{p}) \\
& \quad=\sum_{\mu, \nu, \eta} q^{(a+1) \kappa_{\mu} / 2}(-1)^{|\mu|} W_{\mu, \nu}\left(q^{-1}\right) Q^{|\nu|} q^{\kappa_{\nu} / 2} W_{\nu}\left(q^{-1}\right) \cdot \frac{\chi_{\mu}(\eta)}{z_{\eta} \sqrt{-1}^{l(\eta)}} p_{\eta}(\mathbf{x}) .
\end{aligned}
$$

This is essentially $Z^{-(a+1)}(-\lambda ; t ; \mathbf{p})$, with an extra $(-1)^{|\mu|}$. Hence by Proposition 3.1 , one can easily get:

Proposition 3.2. The normalized open string amplitude of the resolved conifold with one outer brane as in Figure 1(b) and framing $a \in \mathbb{Z}$ is given by:

$$
\hat{\tilde{Z}}^{(a)}(\lambda ; t ; \mathbf{p})=\left\langle\exp \left(\sum_{n=1}^{\infty} \frac{p_{n}(\mathbf{x})}{n \sqrt{-1}} \beta_{n}\right) q^{a K} \exp \left(\sum_{n=1}^{\infty}\left(\frac{1}{[n]}-\frac{e^{-n t}}{[n]}\right) \frac{\beta_{-n}}{n}\right)\right\rangle .
$$

\subsection{Proof of the full Mariño-Vafa Conjecture. Write}

$$
\hat{Z}^{(a)}(\lambda ; t ; \mathbf{p})=\exp \sum_{\mu \in \mathcal{P}^{+}} \sum_{g=0}^{\infty} \sum_{k=0}^{\infty} F_{g ; k ; \mu}^{(a)} \lambda^{2 g-2} e^{-k t} p_{\mu}(\mathbf{x}) .
$$

and

$$
\hat{\tilde{Z}}^{(a)}(\lambda ; t ; \mathbf{p})=\exp \sum_{\mu \in \mathcal{P}^{+}} \sum_{g=0}^{\infty} \sum_{k=0}^{\infty} \tilde{F}_{g ; k ; \mu}^{(a)} \lambda^{2 g-2} e^{-k t} p_{\mu}(\mathbf{x}),
$$

where $\mathcal{P}^{+}$is the set of nonempty partitions. The following is a mathematical formulation of the full Mariño-Vafa Conjecture [13]: 
Theorem 3.1. For the resolved conifold with one outer brane, the generating functions for the open Gromov-Witten invariants are given by:

$$
\begin{aligned}
\exp \sum_{\mu \in \mathcal{P}^{+}} \sum_{g=0}^{\infty} \sum_{k=0}^{\infty} \sqrt{-1}^{l(\mu)} F_{g ; k ; \mu}^{(a)} \lambda^{2 g-2} e^{(|\mu| / 2-k) t} p_{\mu}(\mathbf{x}) & \\
& =\sum_{\mu} s_{\mu}(\mathbf{x}) q^{(a+1) \kappa_{\mu} / 2} \cdot \operatorname{dim}_{q} R_{\mu^{t}},
\end{aligned}
$$

$$
\begin{aligned}
\exp \sum_{\mu \in \mathcal{P}^{+}} \sum_{g=0}^{\infty} \sum_{k=0}^{\infty} \sqrt{-1}^{l(\mu)} \tilde{F}_{g ; k ; \mu}^{(a)} \lambda^{2 g-2} e^{(|\mu| / 2-k) t} & p_{\mu}(\mathbf{x}) \\
& =\sum_{\mu} s_{\mu}(\mathbf{x}) q^{a \kappa_{\mu} / 2} \cdot \operatorname{dim}_{q} R_{\mu} .
\end{aligned}
$$

Proof. It is easy to see from (42) and (46) that

$$
\begin{aligned}
& \exp \sum_{\mu \in \mathcal{P}^{+}} \sum_{g=0}^{\infty} \sum_{k=0}^{\infty} \sqrt{-1}^{l(\mu)} F_{g ; k ; \mu}^{(a)} \lambda^{2 g-2} e^{(|\mu| / 2-k) t} p_{\mu}(\mathbf{x}) \\
& =\left\langle\exp \left(\sum_{n=1}^{\infty} \frac{t^{n / 2} p_{n}(\mathbf{x})}{n} \beta_{n}\right) q^{(a+1) K} \exp \left(\sum_{n=1}^{\infty}\left(\frac{(-1)^{n-1}}{[n]}+\frac{Q^{n}}{[n]}\right) \frac{\beta_{-n}}{n}\right)\right\rangle \\
& \exp \sum_{\mu \in \mathcal{P}^{+}} \sum_{g=0}^{\infty} \sum_{k=0}^{\infty} \sqrt{-1}^{l(\mu)} \tilde{F}_{g ; k ; \mu}^{(a)} \lambda^{2 g-2} e^{(|\mu| / 2-k) t} p_{\mu}(\mathbf{x}) \\
& =\left\langle\exp \left(\sum_{n=1}^{\infty} \frac{t^{n / 2} p_{n}(\mathbf{x})}{n} \beta_{n}\right) q^{a K} \exp \left(\sum_{n=1}^{\infty}\left(\frac{1}{[n]}-\frac{e^{-n t}}{[n]}\right) \frac{\beta_{-n}}{n}\right)\right\rangle .
\end{aligned}
$$

Now we have

$$
\langle 0| \exp \left(\sum_{n=1}^{\infty} \frac{t^{n / 2} p_{n}(\mathbf{x})}{n} \beta_{n}\right)=\sum_{\mu}\langle\mu| t^{|\mu| / 2} s_{\mu}(\mathbf{x}) .
$$

Therefore by using (35) and (34), the proof is completed.

\section{References}

[1] M. Aganagic, A. Klemm, M. Mariño, C. Vafa, The topological vertex, Commun. Math. Phys. 254 (2005), 425-478, arXiv:hep-th/0305132.

[2] M. Aganagic, M. Mariño, C. Vafa, All loop topological string amplitudes from Chern-Simons theory, arXiv:hep-th/0206164.

[3] R. Gopakumar, C. Vafa, On the gauge theory/geometry correspondence, Adv. Theor. Math. Phys. 3 (1999), no. 5, 1415-1443, arXiv:hep-th/9811131.

[4] S. Gukov, A. Schwarz, C. Vafa, Khovanov-Rozansky homology and topological strings, arXiv:hep-th/0412243.

[5] S. Gukov, A. Iqbal, C. Kozçaz, C. Vafa, Link homologies and the refined topological vertex, arXiv:0705.1368.

[6] S. Katz, C.-C. Liu, Enumerative geometry of stable maps with Lagrangian boundary condtions and multiple covers of the disc, Adv. Theor. Math. Phys. 5 (2001), 1-49. 
[7] M. Khovanov, L. Rozansky, Matrix factorizations and link homology, arXiv:math.QA/0401268.

[8] J. Li, C.-C. Liu, K. Liu, J. Zhou, A mathematical theory of the topological vertex, Geom. Topol. 13 (2009), no. 1, 527-621, math.AG/0408426.

[9] C.-C. Liu, K. Liu, J. Zhou, A proof of a conjecture of Mariño-Vafa on Hodge Integrals, J. Differential Geom. 65 (2003), 289-340.

[10] C.-C. Liu, K. Liu, J. Zhou, A formula on two-partition Hodge integrals, Journ. AMS 20 (2007), 149-184,, math.AG/0310272.

[11] J. Li, Y.S. Song, Open string instantons and relative stable morphisms, Adv. Theor. Math. Phys. 5 (2001), no. 1, 67-91.

[12] I.G. MacDonald, Symmetric functions and Hall polynomials, 2nd edition. Claredon Press, 1995.

[13] M. Mariño, C. Vafa, Framed knots at large N, Orbifolds in mathematics and physics (Madison, WI, 2001), 185-204, Contemp. Math., 310, Amer. Math. Soc., Providence, RI, 2002.

[14] A. Okounkov, R. Pandharipande, Hodge integrals and invariants of the unknot, Geometry \& Topology, Vol. 8 (2004), Paper no. 17, 675-699.

[15] H. Ooguri, C. Vafa, Knot invariants and topological strings, Nucl. Phys. B. 577 (2000), 419 438, hep-th/9912123.

[16] E. Witten Quantum field theory and the Jones polynomial, Comm. Math. Phys. 121 (1989), no. 3, 351-399.

[17] E. Witten, Chern-Simons gauge theory as a string theory, in The Floer memorial volume, $\mathrm{H}$. Hofer, C.H. Taubes, A. Weinstein and E. Zehnder, eds., Birkhäuser 1995, 637-678, arXiv:hepth/9207094.

[18] J. Zhou, A conjecture on Hodge integrals, math.AG/0310282.

[19] J. Zhou, Localizations on moduli spaces and free field realizations of Feynman rules, math.AG/0310283.

[20] J. Zhou, Curve counting and instanton counting, arXiv:math/0311237.

[21] J. Zhou, Open string invariants and mirror curve of the resolved conifold, arXiv:1001.0447.

Department of Mathematical Sciences, Tsinghua University, Beijing, 100084, China

E-mail address: jzhou@math.tsinghua.edu.cn 